\title{
Interface Engineering and Direct Bonding of Lithium Tantalate Crystals
}

\author{
ILYA TORCHINSKY ${ }^{1}$ and GIL ROSENMAN ${ }^{1,2}$ \\ 1.-Department of Physical Electronics, School of Electrical Engineering, Tel Aviv University, \\ Tel Aviv 69978, Israel. 2.—e-mail: girl@eng.tau.ac.il
}

A method for modifying the surface free energy of lithium tantalate (LT) ferroelectric crystals is reported. Ultraviolet illumination and low-energy electron irradiation have been used for tuning the surface free energy (wettability) resulting in a wide range of contact angles (6 deg to $87 \mathrm{deg})$. The ultraviolet (UV) illumination makes the LT crystal surface superhydrophilic while the low-energy electron irradiation decreases the surface wetting. Fabrication of various wetting configurations allows demonstration of highquality direct bonding of LT plates with hydrophilic polar faces.

Key words: Direct bonding, ferroelectrics, $\mathrm{LiTaO}_{3}$, surface free energy

\section{INTRODUCTION}

Ferroelectric lithium tantalate (LT) is widely used in optoelectronics due to its unique electro-optical and nonlinear optical properties. In the development and fabrication of high-power nonlinear optical devices based on periodically poled ferroelectrics, direct bonding of several thin crystal plates has proven to be a very promising technique. ${ }^{1}$ Direct bonding means joining of two pieces of the same or different materials without an intermediate layer or external force. The first stage of bonding is adhesion of the sticking surfaces. Adhesion is one of the basic physical phenomena governing gluing, bonding, coating, and many other technologies. It is defined by elementary attractive forces of different nature: van der Waals short-range covalent bonds between adsorbed layers and Lewis acid-base interactions and electrostatic forces of pyroelectric origin acting in ferroelectrics. In order to obtain high-strength adhesive joints, controllable tailoring of the surface free energy and its components (polar and dispersive) is required. Such tailoring could enable significant modification of basic properties related to the surface energy. The latter, such as adhesion and wettability, are critical factors in the bonding mechanism. ${ }^{2}$

(Received January 10, 2008; accepted June 4, 2008;

published online July 2, 2008)
Different techniques have been commonly applied $^{3}$ for attaining two different wettability states: hydrophilic and hydrophobic, providing different mechanisms of bonding. The method of hydrophilic modification produces a hydroxylic surface layer, which forms hydrogen bonds between contacting surfaces during the bonding process. ${ }^{4}$ The concentration of $\mathrm{OH}^{-}$groups at the surface determines the hydrophilicity and may be enhanced using various methods, such as chemical treatment by $1: 1: 6 \mathrm{NH}_{3}: \mathrm{H}_{2} \mathrm{O}_{2}: \mathrm{H}_{2} \mathrm{O}$ solution ${ }^{5}$ or plasma activation. ${ }^{6}$ Recently, superhydrophilicity has been observed in lithium niobate crystals under UV illumination. ${ }^{7}$ On the other hand, hydrophobic modification induces hydrogen- or fluorine-terminated surfaces, ${ }^{8}$ which may be bonded by van der Waals forces between surface $\mathrm{H}$ atoms. ${ }^{9}$

In this paper we report the effect of ferroelectric polar surface modification on direct bonding of LT crystal plates. The LT polar faces have been treated by UV illumination and low-energy electron irradiation. The UV illumination increases the hydrophilicity of the dielectric surfaces ${ }^{7}$ while the low-energy electron irradiation leads to the opposite effect. ${ }^{10,11}$ The Owens-Wendt ${ }^{12}$ and van OssChaudhury-Good ${ }^{13}$ methods of calculating the surface free energy and its components, based on wettability measurements, have been applied. This approach has allowed tailoring and investigating LT 
plate bonding with three different combinations of wetting configurations: hydrophilic/hydrophilic, hydrophobic/hydrophobic, and hydrophilic/hydrophobic.

\section{EXPERIMENT}

Optically polished 0.5 -mm-thick congruent LT crystals of polar cut (Crystal Technology, Inc, USA) were used. The samples were thoroughly cleaned by rinses in acetone, isopropanol, microcleaning detergent, and twice in water for 20 min each in an ultrasonic bath at $50^{\circ} \mathrm{C}$.

Unfiltered light $(\lambda=185 \mathrm{~nm}$ to $2000 \mathrm{~nm})$ generated by a Hg-Xe lamp was used for UV illumination. The illumination duration was around 5 min. Electron irradiation was performed using a specifically designed electron gun (EPG-7, Kimball Physics Inc., USA) in vacuo (base pressure of $10^{-7}$ Torr) at room temperature. The electron irradiation dose was $300 \mu \mathrm{C} / \mathrm{cm}^{2}$ at a constant energy of $E_{\mathrm{p}}=200 \mathrm{eV}$. At this energy, the total electron emission coefficient $\sigma$ of LT is $\sim 1$, thus conserving the irradiated crystal charge neutrality. ${ }^{14}$ The surface potential was measured by Kelvin probe force microscopy, which showed that induced surface charge did not exceed $10^{-12} \mathrm{C} / \mathrm{cm}^{2}$. This value is seven orders of magnitude lower than the spontaneous polarization in LT crystals.

The wettability of the LT samples was estimated by measuring the static contact angles of sessile drops of different probe liquids placed on the LT surface. Optical inspection of the wettability properties was performed by a specially designed system, which combines two charge-coupled-device cameras. The volume of the liquid was kept constant $(2 \mu \mathrm{L})$ for all the contact angle measurements of the different samples. The wettability investigations were carried out with an accuracy of $\pm 1^{\circ}$ at a temperature of $26 \pm 1^{\circ} \mathrm{C}$ and a humidity of $45 \pm 5 \%$. Contact angles were calculated using the Surftens 3.0 software. The values of the surface tension and its polar and dispersive components, for the probe liquids, may be found in Ref. 15. High-resolution x-ray photoelectron spectroscopy (XPS) was used to monitor the chemical surface modification induced by either electron beam or UV illumination. Optical inspection methods of high-resolution atomic force microscopy (AFM) and optical transmittance measurements were used for evaluation of bonding quality.

Three different wettability state configurations were fabricated by the use of combination UV illumination and electron irradiation on polar faces of three pairs of LT plates: hydrophilic/hydrophilic, hydrophobic/hydrophobic, and hydrophilic/hydrophobic. The bonding between LT plates was performed in tail-to-head configuration (where the "tail" is the negative direction of LT spontaneous polarization directions). Such a configuration uses the high natural pressure created by pyroelectric attractive forces generated between the two bonding surfaces when they were primarily heated.

The initial step of the bonding process involves heat treatment at $100^{\circ} \mathrm{C}$ for $\sim 10 \mathrm{~min}$. The generated pyroelectric electrostatic charges of opposite signs pull the two bonding surfaces together and eliminate any remaining voids at the interface. The bonding process is completed by annealing the bonded crystals for $4 \mathrm{~h}$ at $600^{\circ} \mathrm{C}$.

\section{RESULTS AND DISCUSSION}

Figure 1 shows the variation of the static water contact angles at untreated and treated polar faces of the LT plates. The contact angle for the asprepared samples was $\theta=70 \mathrm{deg}$. It decreased to $\theta=6$ deg upon UV illumination. The electron irradiation led to the opposite effect-the water contact angle grew from its initial value to $\theta \sim 87 \mathrm{deg}$.

XPS measurements of the treated samples showed significant variation of the chemical composition and especially of the $\mathrm{C} 1 \mathrm{~s}$ peak intensity. The atomic concentration of carbon on the untreated polar surface was $34 \%$ and varied from $15 \%$ to $82 \%$ under UV and electron irradiation, respectively. Additionally it should be noted that the concentration of oxygen on the surface increased from $44.9 \%$ to $58.3 \%$ under UV illumination and decreased to $16.2 \%$ as a result of electron irradiation. The decrease of $\mathrm{C}$ concentration under UV illumination is attributed to the partial removal of carbon contaminants at the surface due to UV-photoinduced decomposition reaction. ${ }^{16}$ The enhanced hydrophilicity in our experiments may be related to water adsorption at the modified surfaces. Water molecules are adsorbed at the LT surface by dissociation
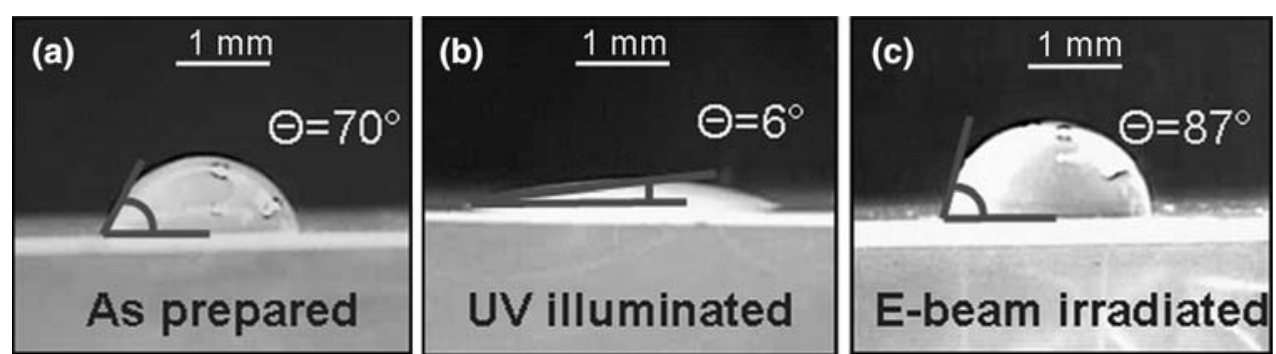

Fig. 1. Variation of the deionized water contact angle at LT surface, induced by various treatments: (a) untreated sample, (b) UV illuminated, (c) E-beam irradiated. 
into hydroxyl groups and protons $\left(\mathrm{H}^{+}\right)$. The hydroxyl group is adsorbed at a surface $\mathrm{Ta}^{5+}$ while the proton forms a second hydroxyl with a surface $\mathrm{O}^{2-}$ ion. ${ }^{17}$ As a result of this chemical reaction, the LT surface is enriched by hydroxyl groups and new layers of water are physisorbed at the surface. The increased oxygen concentration at the surface is explained by simultaneous oxygen and water adsorption at the defective sites. 18

The carbon enhancement found by XPS indicates the formation of a carbon-rich layer at the electron irradiated surface. Hillier ${ }^{19}$ showed that the carbon contamination deposited under electron beam irradiation is formed by the reaction of the incident electrons with organic molecules at the irradiated surface. Such a carbon layer is strongly hydrophobic, ${ }^{20}$ which is consistent with the observed significant growth of the water contact angle for electron-treated samples (Fig. 1).

We studied the surface free energy to understand the wettability variation. The surface free energy may be determined from contact angle measurement by several methods. The Young equation ${ }^{21}$ constitutes a principle for determination of the surface free energy of solids. The analysis and calculation of polar and dispersive components can be performed by the use of the Owens-Wendt equation ${ }^{12}$

$\frac{\gamma_{\mathrm{L}}(\cos \theta+1)}{2\left(\gamma_{\mathrm{L}}^{\mathrm{d}}\right)^{1 / 2}}=\left(\gamma_{\mathrm{L}}^{\mathrm{p}}\right)^{1 / 2} \frac{\left(\gamma_{\mathrm{L}}^{\mathrm{p}}\right)^{1 / 2}}{\left(\gamma_{\mathrm{L}}^{\mathrm{d}}\right)^{1 / 2}}+\left(\gamma_{\mathrm{S}}^{\mathrm{d}}\right)^{1 / 2} \rightarrow y=m x+b$,

where $\gamma$ is the solid surface free energy, and the indices " $\mathrm{d}$ " and " $\mathrm{p}$ " indicate the dispersive and polar (nondispersive) components of the surface free energy, respectively. According to van Oss-ChaudhuryGood ${ }^{13}$ the equation describing the relation between the contact angle and the components of the solid surface free energy is

$$
\gamma_{\mathrm{L}}(\cos \theta+1)=2\left(\gamma_{\mathrm{L}}^{\mathrm{LW}} \gamma_{\mathrm{S}}^{\mathrm{LW}}\right)^{1 / 2}+2\left(\gamma_{\mathrm{L}}^{+} \gamma_{\mathrm{S}}^{-}\right)^{1 / 2}+2\left(\gamma_{\mathrm{L}}^{-} \gamma_{\mathrm{S}}^{+}\right)^{1 / 2}
$$

where $\gamma^{\mathrm{LW}}$ is the dispersive component defined by the Lifshitz-van der Waals interaction and the donor-acceptor term is divided into acceptor and donor polar components, $\gamma^{+}$and $\gamma^{-}$.

The summarized results of the Owens-Wendt calculations (Table I), using measurements with different probe liquids, show that the untreated LT surface free energy is $\gamma=47 \mathrm{~mJ} / \mathrm{m}^{2}$. The components of the surface free energy are $\gamma_{\mathrm{sv}}^{\mathrm{d}}=25 \mathrm{~mJ} / \mathrm{m}^{2}$ and $\gamma_{\mathrm{sv}}^{\mathrm{p}}=22 \mathrm{~mJ} / \mathrm{m}^{2}$. The fraction relation of the polar to the dispersive component is around $\gamma_{\mathrm{sv}}^{\mathrm{p}} / \gamma^{\mathrm{d}}=0.88$. A large contribution of the dispersive component is consistent with the wettability measurements (Fig. 1), showing that the untreated surface of LT is slightly hydrophobic.

The calculated data (Table I) demonstrate that UV illumination strongly modifies the surface free energy of LT by increasing its total value $\gamma_{\mathrm{SV}}$ from $47 \mathrm{~mJ} / \mathrm{m}^{2}$ to $64 \mathrm{~mJ} / \mathrm{m}^{2}$, but the variations of the dispersive and polar components are quite different. While the dispersive component does not change significantly due to the treatment, the polar component strongly increases from $\gamma_{\mathrm{sv}}^{\mathrm{p}} \sim 22 \mathrm{~mJ} / \mathrm{m}^{2}$ to $\gamma_{\mathrm{sv}}^{\mathrm{p}} \sim 44 \mathrm{~mJ} / \mathrm{m}^{2}$, changing the ratio of $\gamma_{\mathrm{sv}}^{\mathrm{p}} / \gamma_{\mathrm{sv}}^{\mathrm{d}}$ to 2.2 . Under UV illumination, the LT surface becomes superhydrophilic with a contact angle of $6 \mathrm{deg}$ (Fig. 1).

Low-energy electron irradiation causes a reduction of the surface energy from $47 \mathrm{~mJ} / \mathrm{m}^{2}$ to $39 \mathrm{~mJ} / \mathrm{m}^{2}$. This is mainly due to a decrease of the polar component from $\gamma_{\mathrm{sv}}^{\mathrm{p}} \sim 22 \mathrm{~mJ} / \mathrm{m}^{2}$ to $\sim 12 \mathrm{~mJ} / \mathrm{m}^{2}$, while the dispersive component increased slightly to $27 \mathrm{~mJ} / \mathrm{m}^{2}$. The ratio $\gamma_{\mathrm{sv}}^{\mathrm{p}} / \gamma_{\mathrm{sv}}^{\mathrm{d}}$ diminishes to 0.44 , resulting in a pronounced hydrophobic state. Thus, the applied UV and electron treatments strongly modulates the surface free energy and its components in the ratio range of $\gamma_{\mathrm{sv}}^{\mathrm{p}} / \gamma_{\mathrm{sv}}^{\mathrm{d}}=2.2$ to 0.44 , which differ by a factor of 5 . This result is consistent with the observed wettability variation from superhydrophilic to pronounced hydrophobic (Fig. 1).

The calculation of surface free energy by the use of another approach (van Oss-Chaudhury-Good) enabled distinguishing between the Lifhsitz-van der Waals (dispersive component) and the nondispersive components. Table II shows the surface free energy variation of LT samples calculated by solving Eq. 2, using different probe liquids.

Table II indicates that a major contribution to the modified surface free energy is due to the electron/ donor term, which varies in the interval $5.9 \mathrm{~mJ} / \mathrm{m}^{2}$ to $50.6 \mathrm{~mJ} / \mathrm{m}^{2}$. The applied UV and electron treatments leads to variation of the surface free energy and its components in the range of polar/dispersive component ratios of $\gamma^{-} /{ }_{\gamma} \mathrm{LW}=1.44$ to 0.12 , which is consistent with the Owens-Wendt estimation (Table II) and the experimental data.

Table I. LT Surface Free Energy and Its Dispersive $\left(\gamma^{\mathbf{d}}\right)$ and Polar $\left(\gamma^{\mathbf{p}}\right)$ Components $\left(\right.$ in $\left.\mathbf{m J} / \mathrm{m}^{2}\right)($ Owens-Wendt Analysis). The Standard Deviation of the Surface Free Energy and Its Component Values Does Not Exceed $2 \%$

\begin{tabular}{|c|c|c|c|c|}
\hline & $\begin{array}{l}\text { Surface Energy } \\
\gamma\left(\mathbf{m J} / \mathbf{m}^{2}\right)\end{array}$ & $\underset{\gamma^{\mathbf{p}}\left(\mathbf{m} \mathbf{J} / \mathbf{m}^{2}\right)}{\text { Polar }}$ & $\begin{array}{c}\text { Dispersive Component } \\
\gamma^{\mathbf{d}}\left(\mathrm{mJ} / \mathrm{m}^{2}\right)\end{array}$ & $\begin{array}{l}\text { Polar/Dispersive } \\
\text { Component Ratio }\end{array}$ \\
\hline Untreated & 47 & 22 & 25 & 0.88 \\
\hline UV illuminated & 64 & 44 & 20 & 2.2 \\
\hline E-beam irradiated & 39 & 12 & 27 & 0.44 \\
\hline
\end{tabular}


Table II. LT Surface Free Energy Electron-Donor Components, $\gamma^{-}$Electron-Acceptor Components, $\gamma^{+}$, and Lifshitz-van der Waals $\gamma^{\mathrm{LW}}$ Components (in $\mathbf{~ m J / m ^ { 2 }}$ ) (van Oss-Chaudhury-Good Analysis). The Standard Deviation of the Surface Free Energy and Its Component Values Does Not Exceed 3\%

\begin{tabular}{|c|c|c|c|c|}
\hline & $\begin{array}{c}\gamma^{-} \\
\left(\mathbf{m} \mathbf{J} / \mathbf{m}^{2}\right)\end{array}$ & $\begin{array}{c}\gamma^{+} \\
\left(\mathbf{m} \mathbf{J} / \mathbf{m}^{2}\right)\end{array}$ & $\begin{array}{c}\gamma^{\mathbf{L W}} \\
\left(\mathbf{m} \mathbf{J} / \mathbf{m}^{2}\right)\end{array}$ & $\underset{\gamma^{-} / \gamma^{\mathbf{L W}}}{\text { Ratio }}$ \\
\hline Initial state & 20.3 & 0.1 & 41.1 & 0.49 \\
\hline UV illuminated & 50.6 & 0.5 & 35.2 & 1.44 \\
\hline E-beam irradiated & 5.9 & 0.18 & 46.2 & 0.12 \\
\hline
\end{tabular}

Ferroelectric LT is an ionic polar crystal which exhibits spontaneous electrical polarization. At equilibrium, the bound charges, which cause spontaneous polarization, are fully screened by compensating charges. These screening charges are localized at impurity levels and surface states near and at a ferroelectric polar face, respectively. The near-surface screening is provided by the electrons and holes of the ferroelectric LT, since it is a semiconductor. $^{22}$ The surface screening occurs due to extrinsic surface states. Our data show that either UV or electron irradiation leads to polar surface modification, changing the surface states concentration and their energy spectrum. Figure 1 shows that UV light makes the surface hydrophilic and cleans it by decreasing the carbon-based contamination at the surface while electron beam irradiation makes the surface hydrophobic and enhances the organic layer. The applied surface modification affects the ratio of the external screening charge and the total compensated charge. For UV-treated LT plates, the surface state concentration (external screening) decreases, leading to growth of the internal screening charge. As a result the nondispersive (polar) component of the surface free energy of UV-treated samples is higher than that for the as-prepared and electron-irradiated samples.

As a test of bonding quality, extreme force (cleavage by a diamond cutter) was applied to the direct-bonded LT crystal pairs until they eventually cracked. Of all three wettability configurations, i.e., hydrophilic/hydrophilic, hydrophobic/hydrophobic, and hydrophilic/hydrophobic, only the first led to reliable direct bonding. Rather than cracking along the direct-bonded interface, the hydrophilic/hydrophilic bonded samples broke in the bulk. These bonded plates exhibited the high interfacial strength required for mechanical processing such as polishing and sawing.

Figure 2 shows AFM and optical images of the direct hydrophilic/hydrophilic bonded interface after a cracking test, demonstrating that the bonded interface looks like an interface of a single grown crystal: it is uniform and void-free. No intermediate layer is observed at the interface and the images are continuous via the boundary between the two LT plates.
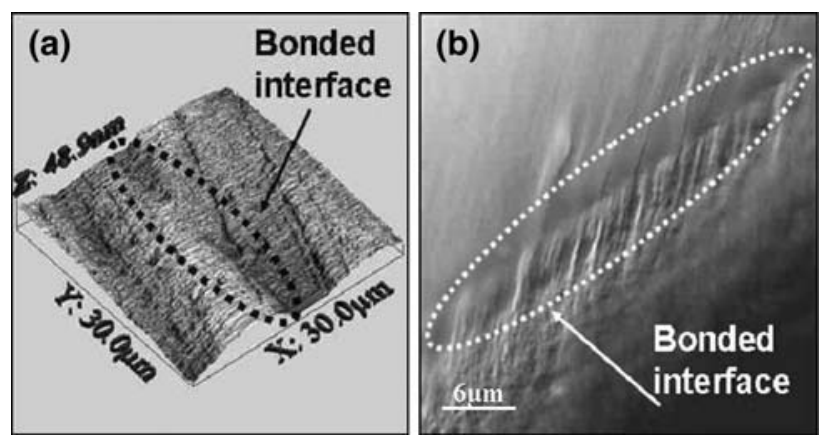

Fig. 2. (a) AFM three-dimensional (3D) topography image and (b) high-resolution optical microscope image of the bonded interface of the cracked direct-bonded $\mathrm{LiTaO}_{3}$.

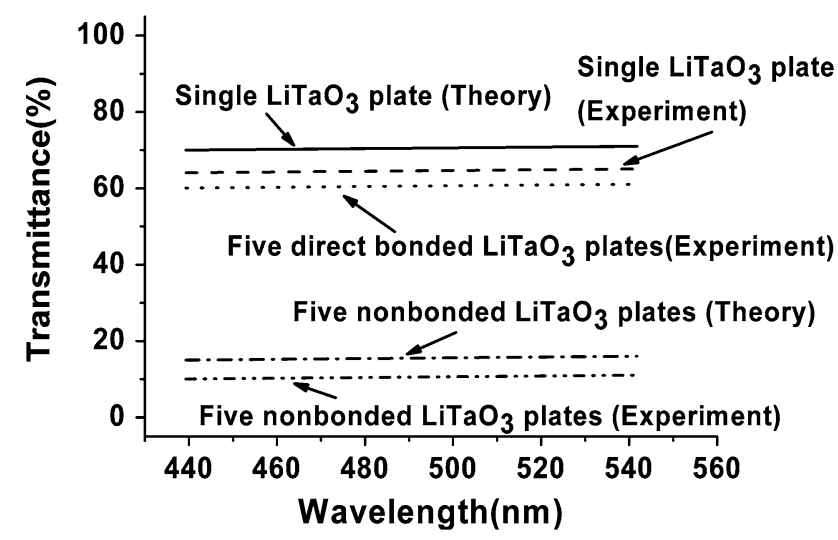

Fig. 3. Optical transmittance characteristics (theory and experiment) of various LT configurations: LT single plate [theory (solid curve) and experiment (dashed curve)], five nonbonded LT plates held together [theory (dash-dot curve) and experiment (dash-dot-dot curve)], and five direct-bonded LT plates (dotted curve).

We fabricated five LT plates with hydrophilic states of their polar faces and bonded them in accordance with the described procedure. The testing of the direct-bonded LT samples was performed using optical transmittance measurements ${ }^{23}$ by comparing a single LT crystal plate, five LT plates pressed together (nonbonded), and five direct-bonded LT plates (Fig. 3). The light beam was launched normally to the polar crystal faces. The graphs were normalized by taking into consideration a measured light intensity level. The theoretical curves (Fig. 3) are based on calculation of transmission losses due to Fresnel reflection at each crystal surface ${ }^{24}$ in this crystal range of transparency. The data showed that the optical losses at each surface of a single LT sample were around $16.5 \%$ (for a wavelength of $450 \mathrm{~nm}$ ) (Fig. 3, theoretical curve). It should be noted that the transmittance changes slightly with the wavelength of the incident light. Additional losses may be due to gaps between the LT plates and voids at the bonded interface. In the case of unsuccessful bonding, optical transmittance should correspond to the nonbonded samples. 
Figure 3 shows theoretical and experimental optical transmittance data for the hydrophilic/ hydrophilic FE configurations. The optical losses at the nonbonded LT interfaces are substantially higher than those for a single crystal. Both theoretical and experimental data of the nonbonded FE plates are consistent. Figure 3 demonstrates that the five direct-bonded LT plates have practically the same transmittance as a single LT crystal, which indicates full merging of the LT plates into a single crystal. These data are consistent with mechanical tests and interface bonding analyses (Fig. 2).

In the process of fabrication of the hydrophilic/ hydrophilic configuration, UV treatment induces a surface layer of $\mathrm{OH}$ groups that, under ambient condition, adsorb a monolayer of water molecules. When polar faces with similar surface energy are contacted at room temperature, bonding is caused by hydrogen bonds between water molecules on the opposing surfaces. On the other hand, direct-bonded hydrophobic stacks are unlikely to provide sufficient interfacial strength and fracture along the directbonded interface under a cracking test. This may be ascribed to the carbon-rich layers that are known to reduce the surface reactivity, eventually preventing covalent bond formation at room temperature. ${ }^{2}$ Also, hydrophobic bonding is susceptible to void formation since gas bubbles may result from hydrogen diffusion to hydrocarbon contamination sites. $^{25}$

\section{CONCLUSIONS}

Thus, controllable surface free energy modification and wettability tuning provide an effective approach for understanding the mechanism of direct bonding and for producing high-quality bonded multilayer ferroelectric devices. The next step in this research is bonding of periodically poled FE crystals and expanding the results to various dielectric and semiconductor materials intended for bonding

\section{ACKNOWLEDGEMENT}

This work was supported by the Israel Science Foundation (Grant No. 960/05).

\section{REFERENCES}

1. M.J. Missey, V. Dominic, L.E. Myers, and C. Robert, Eckardt. Opt. Lett. 23, 664 (1998). doi:10.1364/OL.23.000 664.

2. A. Plößl and G. Kräuter, Mater. Sci. Eng. R25, 1 (1999).

3. M. Alexe and U. Gösele, Direct Bonding: Application and Technology (Berlin: Springer-Verlag, 2004).

4. T. Suni (Ph.D. thesis, Helsinki University of Technology, Finland, 2006).

5. K. Eda, M. Sugimoto, and Y. Tomita, Appl. Phys. Lett. 66, 827 (1995). doi:10.1063/1.113435.

6. M. Nagacubo, S. Fujino, K. Senda, and T. Hattori, U.S patent 5421953.

7. A.C. Muir, S. Mailis, and R.W. Eason, J. Appl. Phys. 101, 104916 (2007). doi:10.1063/1.2734539.

8. K. Ljungberg, A. Wderbarg, and Y.B. Eklund, Appl. Phys. Lett. 62, 12 (1993). doi:10.1063/1.108679.

9. H. Himi, M. Matsui, S. Fujino, and T. Hattori, Jpn. J. Appl. Phys. 33, 6 (1994). doi:10.1143/JJAP.33.6.

10. G. Rosenman, D. Aronov, and Yu. Dekhtyar, PCT patent WO 2007/049280.

11. D.A Aronov, M. Molotskii, and G. Rosenman, Appl. Phys. Lett. 90, 104104 (2007).

12. D.K. Owens and R.C. Wendt, J. Appl. Polym. Sci. 13, 1741 (1969). doi:10.1002/app.1969.070130815.

13. C.J. Van Oss, R.J. Good, and M.K. Chaudhury, Langmuir 4, 884 (1988). doi:10.1021/la00082a018.

14. M.N. Devyatkov, V.I. Ktitorov, and Y.I. Samsonov, Bull. Acad. Sci. USSR, Phys. Ser. (Engl. Transl). 40, 2604 (1976).

15. B. Janczuk, W. Wojcik, and A. Zdziennicka, J. Colloid Interface Sci. 157, 384 (1991). doi:10.1006/jcis.1993.1200.

16. R. Wang, N. Sakai, A. Fujishima, T. Watanabe, and K. Hashimoto, J. Phys. Chem. B 103, 2188 (1999). doi:10.1021/jp983386x.

17. M. Maeda, I. Suzuki, and K. Sakyama, Jpn. J. Appl. Phys. 31, 3229 (1992). doi:10.1143/JJAP.31.3229.

18. X. Feng, L. Feng, M. Jin, J. Zhai, L. Jiang, and D. Zhu, J. Am. Chem. Soc. 126, 6 (2004). doi:10.1021/ja038636o.

19. J. Hiller, J. Appl. Phys. 19, 226 (1948). doi:10.1063/ 1.1715049 .

20. D. Aronov and G. Rosenman, Surf. Sci. 601, 5042 (2007). doi:10.1016/j.susc.2007.09.003.

21. T. Young, Philos. Trans. R. Soc. Lond. 95, 65 (1805). doi:10.1098/rstl.1805.0005.

22. V.M. Fridkin, Photoferroelectrics (Berlin: Springer, 1979).

23. C.B.E. Gawith (Ph.D. thesis, University of Southampton, 2001).

24. J. Wilson and J.F.B. Hawkes, Optoelectronics, 2nd ed. (UK: Prentice Hall, 1989).

25. K.D. Hobart, M.E. Twigg, F.J. Kub, and C.A. Desmond, Appl. Phys. Lett. 72, 1095 (1998). doi:10.1063/1.120975. 